\title{
Aleš Urválek
}

Masaryk-Universität, Brno

DOI: $10.19195 / 0435-5865.141 .7$

\section{„Ich will mir gar nicht alles merken, was mich betrifft." ${ }^{\text {'1 }}$ Autobiographische Texte von H. M. Enzensberger und Peter Schneider}

Es ist kein Geheimnis, dass H. M. Enzensberger für manche 68er inspirativ war, sobald diese ihre eigenen Abhängigkeiten vom Programm der Studentenrevolte abschütteln wollten. Ein kurzer Text von Peter Schneider ${ }^{2}$ zeigt, dass Enzensbergers zunehmender Skeptizismus für Autoren wie P. Schneider in der Tat, so W. Kraushaar, ,eine Art Umerziehungsprogramm, eine Re-education Maßnahme für ehemalige 68er [bot], die sich in ihrem Denken, ihrer Lebenseinstellung, ihrer Mentalität eingemauert haben“. ${ }^{3}$ P. Schneider, elf Jahre jünger als Enzensberger, macht hier keinen Hehl aus seiner Bewunderung für Enzensberger, der die Jugendlichen der 60er Jahre angesprochen habe, indem er „das faustische Suchen, das Kleistische Verzweifeln, das Georgeische Raunen und Gründeln ${ }^{\text {"4 }}$ hinter sich gelassen, und stattdessen Traditionen geltend gemacht habe, die ,seit der Vertreibung und Ermordung der Juden als etwas Fremdes, irgendwie Undeutsches erschienen"5 (Heine, Tucholsky). Am enormen Einfluss Enzensbergers auf die 68er ändert in Schneiders Augen selbst die Tatsache nichts, dass man Enzensberger unter den Studenten sehr selektiv zu lesen pflegte. ${ }^{6}$

Auch die intellektuelle Biegsamkeit von Enzensberger scheint es Schneider angetan zu haben. Gerade weil Enzensberger nichts dabei gefunden habe, „eine frü-

${ }^{1}$ Enzensberger (2014: 105).

2 Schneider (1999: 137-145).

${ }^{3}$ Kraushaar (2010: 61).

${ }^{4}$ Schneider (1999: 138).

5 Schneider (1999: 138).

${ }^{6}$ Der Zorn der ersten zwei Gedichtbände (Verteidigung der Wölfe und Die Landessprache) hatte die nüchternen, sachlicheren und melancholischen Töne des dritten Bandes in den Schatten gestellt, so dass man der Blindenschrift meist keine Beachtung schenkte. 
here, inzwischen überlebte Erkenntnis durch eine neue zu entwaffnen“", 7 imponiert er Schneider derart, dass dieser sogleich der deutschen Meinungsträgheit den Krieg erklären zu müssen glaubt, die eben den dazu bereiten 68ern ihre Meinungsänderung erschwere: den Spieß umdrehend schreibt er, ,in einem Land, in dem das unerschütterliche Festhalten an obsolet gewordenen Überzeugungen als Charakterstärke gilt" ", 8 halte er den Vorwurf der Unzuverlässigkeit „für einen Ehrentitel.“9 Enzensbergers Vorbild scheint Schneider Mut zuzusprechen, er mache nichts verkehrt, wenn er sich von seiner Abhängigkeit von der Neuen Linken freimache, denn ohne diesen gefährlichen Schritt ins Ungewisse wäre er ein lebenslanger Knecht geblieben: „Den Neugierigen unter uns hat er das Denken unter offenem Himmel vorgemacht, den Ausbruch aus dem Denken im Verein, das fröhliche Wildern im Garten des Gegners, die Tugend des Verrats an Überzeugungen, die nur noch Gruppenwärme gewähren“. ${ }^{10}$

Im autobiographischen Text Rebellion und Wahn. Mein 68, den Schneider 2008 verfasst hat, wirkt Enzensbergers Portrait ähnlich hagiographisch, doch allmählich machten sich darin Distanzbemühungen spürbar. Zunächst nimmt Schneider Enzensberger in Schutz, dessen Pech es gewesen sei, ,das jedes, auch jedes unbedachte Wort von ihm kraft seiner enormen Autorität Folgen hatte“, ${ }^{11}$ und da man nicht umhin könne, Fehler zu machen, habe auch er - „wie andere, weniger berühmte Intellektuelle“12 sich geirrt und „Konzepte vertreten, die nicht mehr in sein Selbstbild passen“. ${ }^{13}$ Doch dann wagt er einen „rebellischen“ Wunsch zu äußern, „,man würde gern einen glitzernden Essay von Enzensberger über seine Einlassungen in der Spät- und Verfallszeit der antiautoritären Bewegung lesen, eine Abrechnung mit seinen eigenen ideologischen Verrennungen“" ${ }^{14}$ und dies obwohl, oder eher deswegen, weil „Rückbesinnung und Selbsterforschung “"15 nicht zu seinen Stärken gehören würden. Diese Aufforderung, mit der der Lehrling seinen Meister, der sich nachträglich als (nur) teilnehmender Beobachter „der revolutionären Wirren“ zu stilisieren sucht, unüberhörbar zur Verantwortung zieht, glättet zwar Schneider gleich mit einem neutralen - ,sobald eine alte Überzeugung Risse zeigte, ist er, ohne sich umzudrehen, zu neuen Ufern gebrochen"16 - und (selbst) schützenden Ton - ,aber was ist eigentlich so schlimm an seinen Irrungen und Wirrungen?“ ${ }^{17}$ doch das Distinktionszeichen will er gelten lassen: „zum Bes-

\footnotetext{
${ }^{7}$ Schneider (1999: 144).

${ }^{8}$ Schneider (1999: 144).

${ }^{9}$ Schneider (1999: 144).

10 Schneider (1999: 145).

11 Schneider (2008: 197).

12 Schneider (2008: 197).

13 Schneider (2008: 197).

14 Schneider (2008: 196).

15 Schneider (2008: 196)

16 Schneider (2008: 196)

17 Schneider (2008: 197).
} 
ten, was Intellektuelle zum Fortschritt beizutragen" 18 hätten, würden neben den Erkenntnissen auch Irrtümer gehören, doch man müsse sie vorher eingestanden und intelligent analysiert haben. Folgende Beobachtungen gehen davon aus, dass auf diese durchaus vernünftige Aufforderung Enzensberger in seinen Texten überhaupt nicht eingeht.

\section{Inter- und intragenerationelle Perspektiven}

Den vielen deutschen Intellektuellen, die um und nach 2000 ihre autobiographischen Texte herausgegeben haben, schloss sich 2014 auch H. M. Enzensberger an. Die Aufforderung, eine intelligente Analyse der eigenen Irrtümer vorzulegen, kann man somit recht gut erstens in den Zusammenhang der autobiographischen Texte, und zweitens in das Spannungsverhältnis der Autoren einbetten, die zwei Generationen angehören. Der Generation der sogenannten 45er (geboren 1925 1930) und der 68er (geboren nach 1940). Und sogleich springen einige interessante Tatsachen ins Auge. Haben die meisten Angehörigen der Generation der 45er, also etwa Günter Grass (Beim Häuten der Zwiebel; 2006), Martin Walser (Ein springender Brunnen; 1998), Reinhard Baumgart (Damals; 2003) oder Joachim Fest (Ich nicht; 2006) ihre autobiographischen Texte deutlich auf die Nazijahre hin orientiert, ${ }^{19}$ also aus der Erinnerung heraus zu beschreiben gesucht, wie sie mit der Prägung dieser Jahre umgegangen sind, ist das Gravitationszentrum in Enzensbergers Autobiographie Tumult (2014) um etwa 20 Jahre und viele Tausende Kilometer verschoben. Tumult spielt sich weder im nationalsozialistischen Deutschland, noch an der Front des Zweiten Weltkrieges ab, sondern in den Zügen, in denen Enzensberger das weite Russland in den 60er Jahren bereiste, an amerikanischen Campusuniversitäten, in kubanischen Bars und auf Zuckerplantagen, und immer wieder auf Berliner Straßen, wo die Studentenrevolte der 60er Jahre in vollem Gange war. Bereits dieser erste Vergleich legt die Annahme nahe, Enzensberger glaubt in seiner Autobiographie den Blick nicht auf die Jahre der rechten Diktatur lenken zu müssen, unter der er aufgewachsen war, sondern auf die 60er Jahre, oder anders, auf die Gefahr der roten Diktatur. Nicht seine jugendlichen Makel unter Hitler scheinen ihm ver- oder abarbeitungswürdig zu sein, vielmehr seine viel spätere große - freilich von ihm immer wieder geleugnete Bereitschaft, die Studentenbewegung samt der immer radikaler werdenden neuen

18 Schneider (2008: 197).

19 Zentrale Rolle stellt die Epoche des Nationalsozialismus dar: etwa Baumgart eröffnet die tatsächliche Handlung seiner Autobiographie Damals (2003) mit der Schilderung seiner Erinnerungen an die Ankunft Hitlers in Breslau, wo Baumgart aufwuchs, Walsers autobiographischer Roman Ein springender Brunnen (1998) geht 1945 zu Ende. Grass' Beim Häuten der Zwiebel (2006) fügt dem nur noch die ersten etwa 14 Nachkriegsjahre hinzu. Auch Fest erinnert sich überwiegend an seine Kinder- und Jugendjahre unter Hitler. 
Linken tatkräftig zu unterstützen und die ans Licht tretenden Verbrechen der chinesischen und kubanischen (vietnamesischer oder kambodschanischer) Revolutionäre als etwas revolutionär Unumgängliches in Kauf zu nehmen.

Um das Besondere dieses Textes von Enzensberger zu erfassen, kommt man um den intra- und intergenerationellen Vergleich nicht herum. Denn sosehr Enzensberger generationell zu Walser, Grass oder Baumgart gehört, thematisch ordnet ihn der Text eher der Generation der 68er zu. Somit nimmt Tumult eine Scharnierstellung ein: Enzensberger greift darin kein generationstypisches Thema auf, sondern eher ein von den jüngeren Autoren bearbeitetes. Doch zugleich ist kaum zu übersehen, dass er über dieses Thema nicht wie ein typischer 68er schreibt. Meine Hypothese ist, dass er an das eigentliche Thema der 68er vielmehr wie ein 45er herangeht. Zu überprüfen wäre, ob seine Art, über die 60er Jahre zu schreiben, dem Duktus und der Strategie nahe kommt, mit denen seine Generationsgenossen über ihre Nazikindheit und -jugend geschrieben haben.

Vorwegnehmend seien noch einige Sätze zum Generationsprofil erwähnt: zwischen dem revolutionären Agieren von Enzensberger in den 60er Jahren, und dem, wie diese Generation etwa Helmuth Schelsky, der sie als „skeptische“ bezeichnete, definierte (misstrauisch, höchst unpathetisch, kein Verständnis für revolutionäre Losungen und begeisterten Idealismus, kein Sinn für allesumwerfende Programme; skeptisch und tolerant, wenn wir unter Toleranz die Fähigkeit verstehen, mit den eigenen Schwächen wie auch mit den Schwächen der anderen zu leben ${ }^{20}$ ), finden sich kaum Berührungspunkte. Ohne Tumult lesen zu müssen, weiß man, dass Enzensberger über lange Jahre, mindestens bis in die 70er Jahre hinein, weit davon war, ein Skeptiker zu sein. Es bedeutet jedoch weder, dass es für immer so geblieben ist, noch, dass zwischen dem Enzensberger der 1960er und 2010er Jahre eine Zäsur stehen muss, die manche Forscher herbeibemühen, um zwischen dem frühen nonkonformistisch-linken Kritiker und dem späten konservativ normalisierten und illusionslosen Skeptiker zu unterscheiden. Die erste Frage lautet, ob Tumult in Bezug auf Enzensbergers Entwicklung erkenntnisfördernd gelesen werden kann. Die zweite betrifft das Verhältnis zwischen Schneider und Enzensberger. Die von Schneider formulierte Aufforderung zielte auf moralische Qualitäten, genauer auf Enzensbergers (Un)Bereitschaft, sich nun, also nach 2000, wie auch immer er sich weltanschaulich umpositioniert hatte, den Widerspruch zwischen seiner jetzigen und damaligen Einstellung zuzugestehen und daraus Folgen zu ziehen. Dies macht für den Leser von Tumult den Blick darauf frei, wie dieser Text das Spannungsfeld zwischen dem Horizont des damals lebenden Protagonisten und dem dessen Autobiographen gestaltet, zumal Enzensbergers intellektuelle Leben sich im Zeichen äußerster Dynamik abspielte. Wie geht, könnte also von Schneiders Aufforderung ausgehend gefragt werden, Enzensberger mit offensichtlichen Brüchen und Diskontinuitäten seiner Biografie in der Autobiographie um? Diese

${ }^{20}$ Vgl. Schelsky (1957: 488). 
Spur der inter- und intragenerationellen Perspektive fruchtbar zu machen, heißt Enzensbergers Autobiographie in den intergenerationellen Kontext der autobiographischen Texte von P. Schneider, und in den intragenerationellen Kontext der autobiographischen Texte von Walser, Grass und Baumgart einzubetten. Welche Aspekte des Vergleichs sind dabei besonders zu beachten?

- Welches Bild des Hauptprotagonisten wird von der Erzählinstanz der Autobiographie im Prozess der Vergegenwärtigung des Einstigen produziert? Etwa: wie habe ich damals die Welt wahrgenommen, was habe ich mir damals gedacht, wie entwickelte ich mich?

- Welche Haltung nimmt die Erzählinstanz gegenüber dem Hauptprotagonisten ein? Nimmt sie sich seiner an, distanziert sie sich von ihm, bewertet sie ihn, oder versagt sie sich jede Bewertung? In wie weit und mit welchen Konsequenzen betreiben die analysierten autobiographischen Texte eine Identitätsrekonstruktion?

- Nimmt die Erzählinstanz die Entfernung zwischen den unterschiedlichen Zeithorizonten als Problem wahr, setzt sie dessen Reflexion auch erzählerisch um?

- Welche Analogien und Differenzen lassen sich zwischen den autobiographischen Aussagen über die Nazijahre einerseits, und über die 60er Jahre andererseits innerhalb einer Generation festhalten?

- Welche Analogien und Differenzen lassen sich zwischen den autobiographischen Erinnerungen an dieselbe Zeit (60er Jahre) jenseits der Generationen festhalten, also zwischen Enzensberger und Schneider?

\section{Der intragenerationelle Blick: Autobiographische Texte der „skeptischen“ Generation}

Walsers autobiographischer Roman Ein springender Brunnen setzt erzählerisch die Annahme um, wir würden über die Vergangenheit (inklusive unserer eigenen) nicht frei verfügen. Dessen eingedenk, dass die Art, wie wir unsere Vergangenheit rekonstruieren, nie interesselos sei, erlegt sich Walser eine Art historisierende Entsagung auf, die freilich allenfalls als Wunschprogramm formuliert werden kann: im Sinne des Historismus darauf zu verzichten, die Vergangenheit an den heutigen Maßstäben zu messen, ja sie möglichst interesselos entgegenzunehmen. Um dieses Programm zu verwirklichen, bemüht Walser etliche Analogien, die die Undisponibilität der Vergangenheit belegen sollen (Vergangenheit sei wie der Traumstoff, auf dessen reine Intentionslosigkeit man im Wachzustand nie herankomme; je direkter man sich der Vergangenheit nähere, desto deutlicher begegne man statt ihr dem Motiv, ob dessen man sie aufsuchen wollte usw.) Als Konsequenz ergibt dieses Programm eine Erzählhaltung, die zu den Protagonisten moralisch diskret zu sein sucht. Das Programm der erzählerischen Diskretion (Enthaltsamkeit, Skepsis) geht auf Walsers Unwillen zurück, sich mittels der Figuren seine eigene jetzige Position rechtfertigen zu lassen. 
Auch in der Autobiographie von Grass weigert sich die alte, sich erinnernde Erzählinstanz, an jenen siebzehnjährigen Jungen, der sie am Kriegsende selber war, Maßstäbe anzulegen, die ihre damaligen Möglichkeiten bei weitem übersteigen und vielmehr den Anforderungen der heute gültigen Erinnerungskultur entsprechen würden. Er weigert sich, seine heutige Überlegenheit zur Geltung zu bringen. Diese Überlegenheit (der alte Grass weiß, was der junge damals nicht wissen konnte) auszuspielen, wäre unverdient, ja lächerlich, weil beide existentiell identisch sind. Da der alte Grass nur deshalb mehr weiß, weil er älter als der junge Grass ist, ist für ihn der Grund, auf diesen Vorteil, also auf jegliches moralische Urteil zu verzichten. Die Strategie ist dieselbe wie bei Walser: man lenkt das Augenmerk vom moralischen Profil der Person weg, an deren Jugend man sich erinnert, hin zu der Kompetenz des Autobiographen, dieses moralische Profil zu klassifizieren. Auch Grass also vollzieht in seiner Autobiographie etwas, was man philosophisch als epoché bezeichnen könnte, also die absichtliche Weigerung, Urteile abzugeben, weil der Urteilende nie genug wissen könne, um ein gerechtes Urteil abgeben zu können.

Grass stellt zwar sein autobiographisches Ich jenseits von Gut und Böse, doch er gewährt ihm keinen Unterschlupf; indem er das existentiell identische Ich in zwei Instanzen spaltet, eröffnet er ein Spiel, in dem beide Ichs nicht aneinander herankommen: das jüngere kann sich vor dem älteren nirgendwo verstecken, das ältere kann gegenüber dem jüngeren seine Überlegenheit nicht geltend machen. Diese Überlegenheit kann unmöglich eine moralische sein, weil sie nur dadurch bedingt ist, dass das ältere Ich von Zusammenhängen nicht absehen kann, die dem jüngeren damals unbekannt waren. Das Fazit ist wiederum eine bewusste Resignation auf diese Überlegenheit; Grass' Vorgehen muss daher nicht zwingend als literarisch alibistische Entlastungsstrategie, sondern kann als gewollter Verzicht bezeichnet werden. Verzicht darauf, dem jungen Ich etwas hinzudichten, als auch auf den Genuss, mit ihm nachträglich abzurechnen. Solche Abrechnung wäre allenfalls einer moralisch einwandfreien Instanz zuzumuten, die Grass, selbst im hohen Alter, nicht beanspruchen will.

Literarisch bleibt Grass in der Autobiographie wie auch schon in der Blechtrommel (1959) der Tradition des Antibildungsromans mit vielen Elementen des pikaresken Romans verpflichtet. Diese bringen den Menschen zurück zu seinen natürlichen Grenzen und setzen dies spielerisch um: demonstrierte bereits Oskar Matzerath, dass der Kleingewachsene mehr sehen und wissen kann als alle Erwachsenen, resigniert die Autobiographie auf die Vorstellung, der ältere Mensch müsse klüger, reifer und moralischer sein als der jüngere. Vom moralischen Profil des (jungen) Menschen wird das Augenmerk auf die Kompetenz gelenkt, das Profil überhaupt beurteilen zu können mit dem schon erwähnten Fazit: Skeptische epoché, also Verweigerung des Urteils aufgrund der fehlenden Kompetenz. Dies potenzieren die pikaresken Elemente, sofern sie die moralisch-psychologischen Motivationen der Geschichte in den Hintergrund rücken, und stattdessen 
das Körperlich-Natürliche akzentuieren, also nicht nur den Überlebensdrang des Protagonisten, sondern auch seine beschränkten Möglichkeiten, den kaum durchschaubaren Horizont der Zusammenhänge, in denen er während seiner Jugend zu agieren hatte.

Auf metaphorischer Ebene setzt Grass deutlich lebensweltliche, ja anthropologische Akzente; das Häuten der Zwiebel, das im Bernstein verkapselte Leben, den ewigen Hunger nach Essen, Liebe und Kunst. Die in der Autobiographie schreibend vollzogene Wahrheits(er)findung ist hier ein Prozess, der weder Anfang, noch Ende hat; Wahrheit und Lüge gehen in ihm insofern ineinander über, als das Leben nur in seinen Variationen existieren kann. Dabei wird deutlich, dass die Tradition, in die sich Grass einschreibt, erweitert wird. Zu Rabelais, Grimmelshausen, J. Paul, Camus, oder Döblin, zu denen er sich mehrmals explizit bekannt hat, kommt nun die Traditon der Moralistik mit ihrem wichtigsten Vertreter M. Montaigne hinzu. Eine in Deutschland vergleichsmäßig wenig entwickelte Tradition, ${ }^{21}$ die auf den Menschen durch die Brille seiner „mores“, also seiner lebensweltlichen Üblichkeiten schaut, sich primär dafür interessiert, was er ist, darum wenig mit der Moral, und viel mit dem Leben des Menschen in seiner ,auch unmoralischen Tatsächlichkeit“22 zu tun hat. Eine Tradition, der Blick auf Montaignes Essays beweist es genauso wie auf Grass' Autobiographie, die weit davon ist, dem Leben - so wie etwa in der Augustinschen Bekenntnistradition einen aufsteigenden finalen Charakter unterzulegen, dessen man sich, die eigene Fehlerhaftigkeit rückblickend für eine vorläufige erklärend, im Akt des Schreibens vergewissert. Auch Grass macht keinen Hehl, so H. Friedrich über Montaigne, aus seinen „Verfehlungen, Schwächen, sein[em] Zurückbleiben hinter einer wie auch immer begründbaren Idealität“, ${ }^{23}$ auch er besitzt zugleich die „Klugheit, einstiges Verhalten nicht hinterher zu verurteilen, [da er] weiss, es entstand seinerzeit aus dem unwiederholbaren Zusammenspiel von Gelegenheit und damaliger Beschaffenheit des ich. “24 Freilich, Grass bleibt schulmäßig wohl nach wie vor der Autor der „Reue“, des „Nicht-mehr so sein-wollens“, doch in der Autobiographie aus dem Jahre 2006 scheint dieser Impuls vollkommen verbraucht, in der bisher geschriebenen Fülle der Texte verarbeitet worden zu sein. Die abzuarbeitenden Makel der Jugend werden somit als eine durchaus ernste - also nicht unter des Menschen Würde stehende - Angelegenheit erinnert, die ohne große Bekenntnisund Bußhaltung auskommt.

Weder Walser, noch Grass geben in ihren autobiographischen Texten vor, ihre autobiographischen Protagonisten hätten ein vorbildhaftes Leben geführt. Deren Konformität braucht nicht seitens des Erzählers besonders herausgestrichen wer-

${ }^{21}$ Mehr zur Begriffs- und Entwicklungsgeschichte der Moralistik s. Marquard (1995: 142 $-155)$.

22 Friedrich (1967: 10).

${ }^{23}$ Friedrich (1967: 2015).

24 Friedrich (1967: 2015). 
den, da sie - jedem der heutigen Leser, der weiß, wie man sich zu benehmen hatte - offensichtlich ist. Falls Walser und Grass ihre Figuren vor etwas schonen, dann eben vor diesem Anspruch, in den Augen der heutigen Leser zu bestehen; darum auch die betont subjektive Ambition ihrer Texte, die kaum vergangenheitsbewältigend wirken wollen, da sie die Vergangenheit zu schildern suchen, wie man sie damals (als sie Gegenwart war) wohl empfunden haben mag. $\mathrm{Zu}$ bestehen haben diese Texte (samt den Figuren) lediglich vor den Lesern, die zu akzeptieren bereit sind, dass man nicht direkt zum Widerstand gehören musste, um die Nazijahre ohne Würdeverlust überleben zu können.

Die Erzählperspektive, die gezielt den Blick des während der 30er und 40er Jahre heranwachsenden Hauptprotagonisten kopiert, kann in dieser Hinsicht andere Fragen aufwerfen, als sie nur diejenigen stellen, die hinter ihr eine absichtliche Eliminierung ambivalenter Bilder ob der Entlastung des Erzählers, wenn nicht der gesamten deutschen Nation vermuten. ${ }^{25}$ Ließe man für einen Moment die gesinnungsästhetische Perspektive beiseite, dann wäre nicht mehr zu fragen, warum dieser Junge dem Nationalsozialismus nicht widerstanden hatte, sondern vielmehr, welche reale Möglichkeit er überhaupt hatte (wie jeder Mensch seines Alters), wenn er nicht mitmachen wollte. Einzige Formen des Ungehorsams, von denen etwa in Walsers Text die Rede ist, bestehen darin, dass man den Eltern Gehorsam verweigert, recht egozentrisch zum körperlichen Ungehorsam des „Beichtwürdigen“, wie auch zum geheimen Nonkonformismus der Welt der Buchstaben und Wortbilder flüchtet. Freilich, dass wir all diese Bestrebungen wiederum doppelkodiert lesen können, und zwar entweder als authentische Schilderung des damaligen Lebens jenseits der Illusionen (von eigenem Heldentum), oder andererseits als einen erzählerischen Alibismus des sich selbst entlastenden und seine eigene Feigheit retuschierenden Autors, der seine Jugend schönen will ${ }^{26}$ belegt nur noch, dass man Walsers und Grass' autobiographische Texte aus der Spannung heraus verstehen sollte: ihnen nur anthropologische Bequemlichkeit vorzuhalten, oder sie nur vor geschichtsphilosophischen Zumutungen zu schonen, hieße, dieser Spannung nicht gerecht zu werden.

Gemeinsam haben die autobiographischen Texte dieser Generation, dass sich deren Protagonisten nachträglich keinen politischen Ungehorsam hinzudichten; ungehorsam, also unangepasst war man allenfalls, sofern man leichtfertig und kindisch „egozentrische, privatistische Rückzugsversuche“ ${ }^{\text {27 }}$ unternommen hatte. Die Motivation dazu war selten eine politische, ja eine bewusste; es war meist ein Widerstand am Leitfaden des Leibes. Der Vorwurf, der gegen diese Texte erhoben wird, kommt daher von der anderen Seite: diese Autoren würden sich allzu bequem aus der Affäre ziehen, indem sie alles auf die Karte ihrer unmündigen Unschuld setzen würden.

\footnotetext{
${ }^{25}$ Vgl. Lorenz (2005: 380).

26 Vgl. Baumgart (2003: 10).

27 Baumgart (2003: 128).
} 


\section{Der intergenerationelle Vergleich: autobiographische Texte über die 60er Jahre}

Die Doppelfigur, in der das Körperliche und Politische, Private und Öffentliche verkoppelt und mit der Mündigkeit und Unmündigkeit, mit der Eitelkeit und dem Eskapismus verschränkt werden, weist bereits auf die autobiographischen Texte, die die 60er Jahre thematisieren, also eine Zeit, die als solche unterm Zeichen der politischen und sexuellen Revolution stand, und darum darauf aus war, die Grenzen zwischen dem Privaten und Politischen verschwinden zu lassen.

Schneiders Rebellion und Wahn und Enzensbergers Tumult stemmen sich aus dem Rückblick diesem Gebot der Stunde, indem sie die unheilvollen Konsequenzen sichten, die der Versuch, Politisches und Privates zusammenzuführen, nach sich gezogen hat. Es wimmelt darin nur so von offensichtlichen Widersprüchen: beflügelnden Ambitionen und hohen Idealen der politischen Selbstverwirklichung werden zahlreiche private Katastrophen, trostlose Unfähigkeit, mit Beziehungen zurecht zu kommen, ja revolutionäre Müdigkeit und zunehmende Orientierungslosigkeit, wofür man eigentlich noch kämpfen und wogegen man demonstrieren sollte, gegenübergestellt. Das private Leben der Revolutionäre scheint geradezu aufgesaugt, die Worte dafür ihnen abhanden gekommen zu sein. Man könnte fast sagen, gegen dieses restlose Aufgehen des Privaten im Politischen aufzuschreiben, ist einer der wichtigsten Impulse dieser Bücher, hatte es doch beiden Autoren offensichtlich stark zugesetzt. Darum laufen beide Texte oft darauf hinaus, über die schlichtweg unpolitische, also eher ,allzumenschliche“ Motivation vieler politischer Taten aufzuklären. Weniger aus politischen, vielmehr aus privaten Gründen habe manches resultiert, was sich politisch gegeben habe. Diese autobiographische Aufklärung über die Revolution will besagen, es war weniger Vernunft als Wille, weniger Kopf als Körper, weniger Macht als Ohnmacht, was die Revolution am Leben gehalten hat. Wer Enzensbergers oder Schneiders Rolle in diesen Jahren nur politisch zu erklären und deren Aktivitäten nur als Folgen von rational durchdachten Strategien und Diagnosen zu interpretieren gewohnt war, wird hier eines Besseren belehrt.

Doch diese Figur hat wiederum zwei Seiten; so wie man Walser und Grass zugute halten muss, dass sie ihren jugendlichen Ungehorsam nicht als einen bewussten hochstilisieren, wird man auch Schneider und Enzensberger hoch anrechnen müssen, dass sie ihre großen revolutionären Taten auf ihre ,allzumenschlichen“ Motivationen hinunteraufklären, und den Blick auf Koinzidenzen, Dummheiten, Widersprüchlichkeiten, ja Selbstentlastungen und Selbstbelügungen der Revolutionäre frei machen. ${ }^{28}$ Und wiederum so wie man Walser und Grass hie und da vorhält, sie würden bewusst auf die Karte der jugendlichen Unmündigkeit und politischer Unzurechnungsfähigkeit setzen, um sich von jeder Verantwortung

${ }^{28}$ Vgl. Schneider (2008: 279-280, 293), Enzensberger (2014: 110, 140). 
freizusprechen, könnte man auch die akzentuierte Allzumenschlichkeit für einen künstlichen Regresswunsch von Enzensberger und Schneider erklären, die sich damit der Verantwortung für die eigenen Taten entziehen, und eine jugendliche Unschuld beanspruchen. In diesem Falle wäre dies wohl noch schwerer wiegend, da Enzensberger damals nicht 17 wie Grass oder Walser war, sondern fast 40 Jahre alt war.

Welche Entwicklungslinie der Hauptprotagonisten wird in Schneiders und Enzensbergers Texten gezeichnet? In wie weit wird in ihnen die Geschichte darüber erzählt, wie man das geworden ist, was man nun zu sein glaubt? Schneiders Schilderung seiner Kinder- und frühen Jugendjahre in Freiburg hält sich an typisierte Abbildung der bundesrepublikanischen 1950er Jahre; Freiburg steht für all das Restaurative, viele Rückzugsmöglichkeiten ins Existentialistische und Esoterische Bietende, die Vergangenheit Beschweigende, und kulturbeflissen Idealisierte, in das der junge, eckige, nervöse, gegen alles Runde allergische Schneider wunderbar passt, um es bald als spießige Provinz hinter sich zu lassen. Doch mit der Geburt eines wirklichen Revolutionärs, der mittlerweile in Berlin zu agieren beginnt, will es nicht ganz klappen, ständig kommt ihm seine Feigheit in die Quere. Sie wirkt auf den ersten Blick neben seinen hypermutigen Zeitgenossen recht sympathisch, sie macht aus dem Revolutionär einen Menschen, zumal Schneider wahrlich nicht wenig zu berichten hat von seinen meist aus Feigheit begangenen revolutionären Dummheiten. Beteiligt hat er sich an ihnen, so beteuert er nun in der Autobiographie, weil ihm einfach Mut gefehlt habe, ,gegen die überschnappenden Führer in den eigenen Reihen zu protestieren“. 29

Man sieht, Schneider spannt seinen Protagonisten zwischen zwei Mut- bzw. Feigheitsbegriffe ein: ihm fehlte es damals an Mut, ,gegen die Führer in der eigenen Gruppe aufzustehen und zu sagen: Ihr spinnt! Ihr seid verrückt geworden!“30 Genauso mutlos war er aber auch, als es hieß, sich als wirklicher Revolutionär zu bewahren, der keine Gewalt scheut. Als damaliger Akteur wollte er die zweite Mutlosigkeit überwinden, um nicht nur Plakate und Reden entwerfen zu müssen, sondern auch in den ersten Reihen zuschlagen zu können, dort verprügelt zu werden, was ihm dann trotz allen Hemmungen gelungen ist. ${ }^{31}$ Als Autobiograph schämt er sich rückblickend viel stärker der ersten Mutlosigkeit (dass er einfach weiter mitmachte, obwohl er längst gerne aufgehört hätte), und darum - dies wohl kompensierend - stilisiert er seine damalige Mutlosigkeit, sich an Gewaltaktionen zu beteiligen, zum großen Teil als eine bewusste humanistisch konditionierte Absage. Wer bei den Demonstrationen keine Steine warf, dies geht deutlich aus der Szene mit der schmalschultrigen blonden Demonstrantin „mit Kurzhaarschnitt“ hervor, war einfach feige, ein verklemmter Versager in puncto Politik und Männlichkeit, der nur falschen bürgerlichen Hemmungen gehorchte. Doch wer Steine gegen die

\footnotetext{
${ }^{29}$ Schneider (2008: 175).

${ }^{30}$ Schneider (2008: 362).

31 Vgl. Schneider (2008: 130-131).
} 
Schaufenster und Polizisten warf, obwohl er Bedenken hatte, war genauso mutlos und feige, nämlich der Brutalität Einhalt zu gebieten. Was die Erzählinstanz der Autobiographie nun gut weiß, hat der Hauptprotagonist damals allenfalls geahnt, ohne sich danach konsequent gerichtet zu haben. Die Folgen dieser kompensatorischen Stilisierung bekommt die Blondine zu spüren, die mit ihrem militaristischen Wortschatz (,Was ist los mit dir, Ladehemmung?“32) schlecht wegkommen muss, da die Erzählinstanz betont, dass die Revolutionärin über den ausschlagegebenden Unterschied zwischen (Gewaltanwendung gegen) Sachen und Menschen hinwegsah, und somit die ganze Revolution nur zum Revival der Totalität machte. $\mathrm{Zu}$ dieser Einsicht, und dies scheint mir wichtig zu sein, gelangte Schneider erst im nachhinein, doch er kann nicht umhin, sie für seine damalige Sicht zumindest teilweise zu reklamieren. Sein junges Ich wird ihm geradezu zum Kontrapunkt der militaristischen Blondine: schon beim Anblick eines Polizisten will er Unbehagen bei der Vorstellung empfunden haben, der Stein könne das Gesicht eines Menschen treffen; die Fähigkeit, hinter dem Polizisten einen Menschen zu sehen, ist jedoch den damaligen Revolutionären vollkommen abhanden gekommen; durch diese stilisierte Schilderung erhebt sich Schneider über die Revolutionäre desto massiver, je unfähiger (da mutlos) er dazu damals gewesen war.

Die Mutlosigkeit, die wohl den zentralen Punkt der Textes bildet, schlägt sich in ihm darin nieder, dass die Erzählinstanz recht unstabil hin und her wankt: manchmal wird das junge Ich zu einem feigen Menschheitshelden stilisiert, anderes Mal neigt die Erzählinstanz dazu, mit den damaligen Revolutionären, inklusive sich selber, regelrecht abzurechnen; ${ }^{33}$ doch in beiden Fällen macht sie eben seinen Wissens- und Altersvorsprung geltend, um an ihm entweder das junge Ich (unverdient) partizipieren zu lassen, oder es schlichtweg zu distanzieren. Somit droht der Text von Schneider die Grenze zu überschreiten, an der das verdienstvolle Vermenschlichung der Revolutionäre ins fanatische Abrechnen, oder aber in deren Entlastung umkippen kann. Damit wird langsam deutlich, wie Schneiders Autobiographie die Identität des erzählten Lebens konstruiert. Das junge Ich wähnte sich identisch, doch es war, wie die Erzählinstanz nun weiß, eine falsche Identität: „Wie konnte der Schreibende vor vierzig Jahren diesen Abgrund überspringen“, 34 bemerkt er bitter mit Blick auf den offensichtlichen Widerspruch zwischen ,priva-

32 Schneider (2008: 129).

33 Seine damaligen Reaktionen befremden die Erzählinstanz vollends, beim Kommentieren seiner damaligen Tagebucheintragungen rechnet er mit sich selbst ab. Rückblickend schildert sich Schneider inmitten der Revolutionäre so, als wären sie damals alle in einem Spiel, das etwas Traumhaftes, Schizoiden und Unbeholfenes hatte (revolutionäre Rollen und Aufgaben werden fast wie bei der Laiengruppe im Sommernachtstraum von Shakespeare verteilt) und das man unfreiwillig im Gange hielt, da man nicht aussteigen konnte (Wer auf Fragwürdigkeit des Spiels hinweist, wird gleich gruppendynamisch als Kleinbürger, Klassenfeind usw. disqualifiziert). Die Handlung wird in der psychiatrischen Sitzung abgerundet, die ja nur den schizoiden Traumwahn der Revolution ins Reale transponiert.

${ }^{34}$ Schneider (2008: 14). 
ter Verzweiflung und der Siegeszuversicht bei den Demonstrationen“35 in seinen damaligen Tagebucheintragungen. Nun gilt es, auf dem Weg der Reflexion die falsche Identität hinter sich zu lassen und eine wahre zu erreichen. Dies geschieht um den Preis, dass die Erzählinstanz die Welt des jungen Ich in Frage stellt. Somit schreibt sich Schneider in das Muster ein, das um der jetzigen Identität willen die früheren Lebensphasen zu definitiv unwahren herabsetzen muss. Der jetzige wahre Stand der Idealität wird von den früheren Lebensphasen durch einen Wendepunkt getrennt, von dem an sich jede Zweifel erübrigen.

Wenn also Schneider, um zu der einführenden Fragestellung zurückzukehren, von Enzensberger eine intelligente Abrechnung mit seinen Irrtümern erwartet, denkt er wohl an eine derartige Selbstabrechnung, an das, rein typologisch, Augustinische Modell der Bekenntnisautobiographie, freilich in säkularisierter Form. Denn zu diesem Modell fehlen in Schneiders Autobiographie wahrlich keine Ingredienzen: man hat sowohl den Wendepunkt, mittels dessen sein Ich zu seiner idealen Selbstvollendung wird, als auch den finalen Charakter, der dem Subjekt das Recht gibt, auf das Geschehene bereits vom Gipfel der Erfüllung zurückzublicken, auch die Zweifel, die indes allenfalls dazu da sind, um letztendlich überwunden zu werden. Diesen Erwartungen kommt Enzensbergers Autobiographie nicht nach, ihr Modell scheint sich weniger an der moralischen Bekenntnistradition vom heiligen Augustin, vielmehr an der Montaigneschen Tradition der Moralistik und Skepsis zu orientieren. Die Autobiographie Tumult wie auch die Biographie Hammerstein oder Eigensinn ${ }^{36}$ legen davon einen klaren Beweis ab. In beiden steht im Mittelpunkt eine recht eigensinnige Figur: der General Kurt von Hammerstein (1878-1943) und H. M. Enzensberger, beide spielen sich in umstrittenen und kontroversen Jahren ab: um 1933 und um 1968. Entscheidend scheint mir an diesen Texten, die beide panoramatisch den Blick auf rechte und linke Totalitätsversuchungen frei machen, das $\mathrm{Ma} ß$ der Freiheit, das sie ihren, beileibe nicht vorbildhaften ${ }^{37}$ Helden gönnen. Beide Texte machen das Recht der Figuren auf Eigensinn geltend, um sie von der Allmacht der ideologischen Schemen zu befreien. Denselben Eigensinn, den Enzensberger Hammerstein und dessen Kindern einräumt, beansprucht er selbst mit der Folge: je eigensinniger die Figuren, desto weniger kann die Erzählinstanz auf sie herabblicken. Warum Hammerstein seine Verachtung der Nazis in keine politisch konsequente Widerstandsaktion umge-

35 Schneider (2008: 14).

${ }^{36}$ Enzensberger (2008).

${ }^{37}$ K. Hammerstein, dem obersten Offizier der Reichswehr, einem konservativen General, könnte man etwa vorwerfen, dass er in der ersten Hälfte der 30er Jahre, als es noch möglich war, und er dazu sicher genug Gelegenheiten gehabt hatte, kein klares Nein zu Hitler gesagt hat, obwohl er ihn sehr kritisch sah. Und Enzensberger könnte man vorwerfen, dass er sich lange seine Illusionen über die linke Revolution nicht nehmen ließ, sogar nach Kuba ging, um die Revolution live zu erleben und zu unterstützen; vorzuwerfen wäre ihm weiter, dass er, statt einzusehen, dass hier auf dem Weg zur freien Gesellschaft vollkommen unfreie Verhältnisse in Kauf genommen wurden (Lager für Regimekritische, Hinrichtungen, Zensur usw.), diese sozialistischen Experimente mitmachte, ja sogar die sich radikalisierende Linke in Deutschland gelten ließ. 
setzt habe, ist zwar eine berechtigte Frage, aber nicht für Enzensberger. Er entsagt allen Urteilen, bleibt zu seinen Figuren diskret, um hervortreten zu lassen, dass das Leben nicht auf die Wahl zwischen Entweder-Oder zu reduzieren ist. Hammerstein war weder Held, noch Verräter. Und darum auch jemand, der über seine Vergangenheit schweigen kann, ohne sich dem Verdacht aussetzen zu müssen, er verschweige etwas.

Dass es wenig Sinn macht, davon auszugehen, der später Geborene wisse, was man besser hätte machen sollen, weil er automatisch über einen ihn privilegierenden Wissensmehrwert verfüge, demonstriert Enzensberger an seinen in der Hammersteinbiographie verstreuten Totengesprächen, den postumen Unterhaltungen. Hier ist es genau umgekehrt, als man erwarten würde: auch wenn man heute einiges wissen mag, was diese bereits Verstorbenen zu ihren Lebzeiten nicht gewusst haben, ziehen die Heutigen in allen diesen Konfrontationen immer den Kürzeren. Obzwar Tumult ohne das verfremdende und verunsichernde Mittel der Totengespräche auskommt, ändert sich an der Situation kaum etwas. So sehr Enzensberger 2014 weiß, was er in den 1960ern hätte machen müssen, er weiß einfach nicht genug, um sich gegenüber dem damals Lebenden einseitig ins Recht setzen zu können.

Stellen wir Schneiders und Enzensbergers Autobiographie nebeneinander, um deren Titel zu interpretieren. Indem Schneider seinen Text als Rebellion und Wahn, Mein 68 bezeichnet, gibt er deutlich zu verstehen, bemüht sei ein ausgeglichener Blick auf 1968, der Verdienste (nützliche Rebellion gegen den Gehorsamszwang) und Verluste (man hätte sich viel früher besinnen müssen) auf die Waagschale wirft; bemüht sei weiter eine Identitäts(re)konstruktion, in der ein heute 68jähriger sich qua 1968 seiner selbst vergewissert. Da, wo Schneider einen freilich umstrittenen, doch fest verankerten Begriff verwendet, steht bei Enzensberger das Wort Tumult, das im übrigen auch bei Schneider oft vorkommt, aber es nie zum Titel bringt. Damit gibt Enzensberger zu verstehen, bemüht sei weniger Begrifflichkeit, als vielmehr ein offener, chaotischer Entwurf, zumal kein von besonderem Identitäts- und Selbstvergewisserungswunsch geleiteter. Enzensberger tut, nicht viel anderes als etwa Montaigne, alles, um sein Desinteresse an sich selbst, an seiner Biographie zu zeigen. Der Duktus der Prämissen, in denen er über die Motivation seiner Autobiographie schreibt, ist voll dilettantischer Selbstironie („Mein Gedächtnis gleicht einem Sieb, in dem wenig hängen bleibt [...] Allerdings lässt mein Interesse an einer Autobiographie zu wünschen übrig. Ich will mir gar nicht alles merken, was mich betrifft"), ${ }^{38}$ die man eben von Montaigne kennt, der sich auch gerne den Anschein gibt, ein schlechtes Gedächtnis zu haben und in seiner Bibliothek (wie Enzensberger in seinem Gedächtnis) ,wahllos herumblättert und die Zufallsfunde je nach Laune niederschreibt oder vergisst" ${ }^{39}$ Als ein schlechter Adept für eine wahrheitsuchende Autobiographie, ist Enzensberger also bereit, nur

\footnotetext{
${ }^{38}$ Enzensberger (2014: 105)

39 Friedrich (1967: 36).
} 
das von der Hand zu geben, worin Zufall, Chaos und Offenheit regieren, um nur nicht aus großem Abstand von sich selbst erzählen zu müssen. Diese zu Schneider diametral entgegengesetzten autobiographische „Ambition“ ergibt auch gegensätzliche Haltung der Erzählinstanz zu dem jungen Ich; sie lässt keinen Raum für jegliche Selbstrechtfertigung und selbstrevidierende Bekenntnisse offen. So sehr Enzensberger aus seinen damaligen Fehlern keinen Hehl macht, so wenig spürt man bei ihm - auch diesbezüglich wäre man bei Montaigne fündig - das Bedürfnis, sich ob des Zurückbleibens hinter der Idealität ethisch zu aktivieren, sich zu zerknirschen, ja „das nicht mehr so sein wollen“40 des nach Erlösung Drängenden.

Die Wechselbeziehung zwischen der Erzählinstanz und dem jungen Ich entspricht dem Motto: Mit seinen Problemen muss jeder selber fertig werden. Beide sind durch keinen Wendepunkt getrennt, der einen von ihnen umso falscher zeigen würde, je wahrer sich der andere geben könnte. Habe man damals Fehler gemacht, werde man sie wohl auch jetzt machen, Zweifellosigkeit könne keiner von ihnen beanspruchen. Weder die eines damals definitiv falsch Handelnden, noch die eines zu dieser Einsicht Legitimierten. Wollte Schneider die falsche Identität seines jungen Ich in einer wahren auflösen, die er rückblickend bemühte, verzichtet Enzensberger offensichtlich auf diesen Identitätswunsch, um ihm die Freiheit der beiden nicht opfern zu müssen. Auf eine sehr wohl durchdachte Art stellt Enzensberger dies in den Kapiteln „Erinnerungen an einen Tumult“ dar, wo er die beiden Instanzen seines Ichs aufeinandertreffen lässt. Und von vorne ist klar, diese Begegnung sei auf keinerlei Entlastung aus, dafür kennen die beiden einander allzu gut. Sie können sich weder etwas einreden, noch etwas vormachen, die Karten sind sehr gerecht verteilt. Ein ungeahntes Gleichgewicht scheint hier zu walten. Der Alte durchschaut jedes Alibi des Jungen. Der Junge lässt sich nicht herablassend behandeln. Sie lassen sich voneinander weder missbrauchen, noch entlasten. Der Alte ist weder imstande, dem Jungen etwas vorzuwerfen, noch bereit, ihm eine Absolution zu erteilen. Er geht auf ihn nicht mit der moralischen Vehemenz eines einstigen Revolutionäres, der es heute besser wissen will, sondern er will lediglich wissen, was er sich damals „,bei alledem gedacht ${ }^{\text {“41 }}$ habe. Die Spaltung in zwei Ichs unternimmt Enzensberger nicht, um einen auf Kosten des jeweils anderen zu privilegieren (entlasten) oder herabzusetzen. Es gibt hier also keine Möglichkeit für die beiden, sich dem jeweils anderen drunter und drüberzustellen. Jedem von ihnen bleibt nichts anderes übrig, als mit seinen Problemen selber zu Recht zu kommen.

Dessen eingedenk, dass man in keinem Augenblick genug weiß, um ein gerechtes Urteil zu treffen, und dass jedes Moralisieren - der anderen wie auch sich selbst, wie in dieser autobiographischen Spaltung zu sehen war - letztendlich auf eine Entlastung hinausläuft, weist Enzensberger das bekenntnishafte Modell der Augustinischen Autobiographie, an das sich Schneider anlehnt, zurück, und neigt idealtypisch

\footnotetext{
${ }^{40}$ Friedrich (1967: 214).

${ }^{41}$ Enzensberger (2014: 107).
} 
zu demselben Modell, das auch Grass und Walser in ihren autobiographischen Texten bevorzugen. Zugleich ist kaum zu übersehen, so paradox es auf den ersten Blick anmutet, dass all diesen Texten ein Gestus eigen ist, der eigentlich ein versöhnender, mit menschlichen Bedürftigkeiten und Grenzen abgefundener ist. Also ein Gestus, der Verständnis für die Fehler der anderen wie auch seine eigenen zeigt, und eben darum immer zu Skepsis tendiert, wenn er dazu gedrängt wird, definitive Urteile auszusprechen. Damit gelangt Enzensberger nicht nur in enge Verwandtschaft zu seinen Generationsgenossen, die zu genau diesem Gestus in ihren die Nazikindheit und -Jugend thematisierten Autobiografien gefunden haben, sondern auch zu deren skeptischen Ausprägung: gegenüber den eigenen und fremden Schwächen auch dann tolerant zu sein, wenn man dadurch nichts gewinnt.

\section{Literatur}

Baumgart, Reinhard: Damals. Ein Leben in Deutschland. München 2003.

Enzensberger, Hans Magnus: Hammerstein oder Eigensinn. Eine deutsche Geschichte. Frankfurt am Main 2008.

Enzensberger, Hans Magnus: Tumult. Frankfurt am Main 2014.

Fest, Joachim: Ich nicht. Erinnerungen an eine Kindheit und Jugend. Hamburg 2006.

Friedrich, Hugo: Montaigne. Bern 1967.

Grass, Günter: Beim Häuten der Zwiebel. Göttingen 2006.

Kraushaar, Wolfgang: Vexierbild. Hans Magnus Enzensberger im Jahre 1968. In: Petersdorff, Dirk (Hrsg.): Hans Magnus Enzensberger und die Ideengeschichte der Bundesrepublik. Heidelberg 2010. S. 45-64.

Lorenz, Matthias N.: ,Auschwitz drängt uns auf einen Fleck“. Judendarstellung und Auschwitzdiskurs bei Martin Walser. Stuttgart - Weimar 2005.

Marquard, Odo: Der Mensch Diesseits der Utopie. Bemerkungen über Geschichte und Aktualität der philosophischen Anthropologie. In: Marquard, Odo: Glück im Unglück, Philosophische Überlegungen. Leipzig 1995. S. 142-155.

Schelsky, Helmut: Die skeptische Generation. Eine Soziologie der deutschen Jugend. Düsseldorf 1957.

Schneider, Peter: Bildnis eines melancholischen Entdeckers. In: Wieland, Rainer (Hrsg.): Der Zorn altert, die Ironie ist unsterblich. Über Hans Magnus Enzensberger. Frankfurt am Main 1999. S. 137-145.

Schneider, Peter: Rebellion und Wahn. Mein 68. Köln 2008.

Walser, Martin: Ein springender Brunnen. Frankfurt am Main 1998.

\section{Abstracts}

Der Beitrag stellt die autobiographischen Texte von H. M. Enzensberger und P. Schneider nebeneinander, um die Übergänge zwischen der Semantik der sogenannten skeptischen Generation (Autoren, die zwischen 1925-1930 geboren sind, also G. Grass, M. Walser, R. Baumgart, J. Fest) und der Generation des Jahres 1968 (hier P. Schneider) zu ermitteln. Er geht der Aufforderung von Schneider nach, man sei verpflichtet, die eigenen früheren Irrtümer einzugestehen, und versucht zu bergrün- 
den, warum Enzensberger auf diese Aufforderung in seinen Texten überhaupt nicht eingegangen ist. Die Analyse bettet die Enzensbergersche Autobiographie Tumult in den Kontext der intra- und intergenerationellen Perspektive ein, um die Hypothese zu überprüfen, in wie fern Enzensberger an das Jahr 1968 mit derselben Erzählstrategie herangeht, mit der seine Generationsgenossen über ihre Jugendjahre unter Hitler geschrieben haben.

Schlüsselwörter: Autobiographie, Generation, Skepsis, Moralisieren, Bekenntnisse

\title{
„Ich will mir gar nicht alles merken, was mich betrifft.” Autobiographical texts of H. M. Enzensberger and P. Schneider
}

This study compares the autobiographical texts of H. M. Enzensberger and P. Schneider and tries to describe transitions between semantics of the so-called sceptical generation (authors born in 1925-1930, here G. Grass, M. Walser, R. Baumgart, J. Fest) and the '68 generation. It is based on Schneider's proclamation that an intellectual is obliged to admit to their former errors and mistakes. Further it tries to clarify why Enzensberger was not going to fulfil this proclamation in his texts. This analysis puts Enzensberger's autobiography Tumult (2014) in the intragenerational and intergenerational perspective. The main aim of this study is then to prove to what extent Enzensberger's narrative strategy pairs with the strategy of his generation peers writing about their apprenticeship years under the rule of the Nazis.

Keywords: autobiographical texts, Enzensberger, Paul Schneider

\author{
Aleš Urválek \\ Masaryk-Universität Brno \\ Ústav germanistiky FF MU Brno. \\ Arne Nováka 1, \\ 60200 Brno \\ Tschechische Republik \\ E-Mail: urvalek@phil.muni.cz
}

